\title{
ANIMACIÓN DE ROBOT Y FIGURAS HUMANAS
}

\author{
Andrés Morales C. ${ }^{1} \quad$ María Cecilia Rivara ${ }^{1}$ \\ Recibido el 3 de marzo de 2004, aceptado el 20 de diciembre de 2004
}

\begin{abstract}
RESUMEN
En este trabajo se discute un enfoque heurístico al modelamiento del cuerpo humano y su animación. En la literatura técnica se han publicado trabajos respecto a la animación de figuras [1], [2], [3] y en algunos [4], [5] en los que se usan herramientas físicas, tales como el momento de inercia y masa para realizar las animaciones. Los movimientos mostrados en este proyecto, en cambio, muestran una forma alternativa de animación basada en la velocidad de desplazamiento y la amplitud de oscilación de las piernas sin necesidad de usar masas y en consecuencia momentos de inercia.

Específicamente, este trabajo se divide en dos partes. La primera corresponde a la animación de un robot, y la segunda, a la animación de una figura humana motivada por las observaciones y resultados obtenidos de la primera parte.

El método propuesto en la primera parte se separa en tres etapas: el movimiento de piernas, brazos y del robot en su totalidad. Luego estas tres partes se combinan mediante heurísticas y se ajustan para obtener un movimiento armónico y creíble.

En la segunda parte se desarrolló un método que permite implementar los resultados de la primera parte, agregando la funcionalidad de incorporar nuevas partes al modelo (pies, manos, etc...). Se desarrolló una función que a partir de parámetros como la amplitud de oscilación y velocidad de desplazamiento permite recrear el movimiento en forma automática.

Para ambas partes fue necesario construir una aplicación especial para implementar los modelos y observar los resultados. El software desarrollado fue creado usando el lenguaje de programación Java versión JSDK1.4 y la API Java3D para el desarrollo 3D.
\end{abstract}

Palabras clave: Computación gráfica, animación de figuras humanas, animación.

\section{ABSTRACT}

In this work one discusses to a heuristic approach to the modeling of the human body and its animation. In technical Literature works have been published related to animation of figures [1], [2], [3] and some ones [4], [5] in which physical tools are used, such as the moment of inertia and mass to make the animations. The movements shown in this project however, they show to an alternative form of animation based on the speed of displacement and the amplitude of oscillation of the legs with no need to use masses and consequently moments of inertia.

Specifically, this work is divided in two parts. First, it corresponds to the animation of a robot, and second, to the animation of one it appears human motivated by the observations and obtained results of the first part.

The method proposed in the first part, one separates in three stages: the movement of legs, arms and of the robot in its totality. Soon these three parts are combined by means of heuristic and they adjust to obtain a harmonic and credible movement.

In the second part, a method was developed that allows to implement the results of the first part, adding the functionality to incorporate new parts to the model (feet, hands, etc...). Development a function that from parameters as the amplitude of oscillation and speed of displacement allows to recreate the movement in automatic form.

For both parts, it was necessary to construct a special application to implement the models and to observe the results. Developed software was created using the programming language Java version JSDK1.4 and the API Java3D for the development $3 D$.

Keywords: Computer graphics, human figure animation, animation.

\footnotetext{
1 Universidad de Chile, Departamento de Ciencias de la Computación, Avenida Blanco Encalada 2121, Tercer Piso, Santiago Chile, amorales@dcc.uchile.clmcrivara@dcc.uchile.cl
} 


\section{INTRODUCCIÓN}

Una de las áreas de investigación más importantes desde los comienzos de la computación gráfica ha sido el modelar y animar cuerpos o figuras móviles y simular en forma realista algún comportamiento de ellos desde una figura geométrica, como un cubo girando en torno a un eje, hasta formas mucho más complejas, como un ser humano y sus movimientos.

La dificultad de hacer una animación creíble se puede comparar con la anatomía del objeto a modelar; por ejemplo, una figura geométrica como un cubo se puede modelar matemáticamente en forma muy rápida y fácil, sin embargo, la anatomía humana es mucho mas compleja debido a la cantidad de partes móviles y la forma en que sus movimientos dependen a su vez de otras partes del cuerpo. Un punto intermedio entre las figuras geométricas fáciles de modelar y el cuerpo humano es el modelamiento de este último usando figuras geométricas.

Este punto intermedio puede ser más cercano al lado del cuerpo humano; mientras más figuras formen el modelo o más cercano al lado geométrico mientras menos figuras se utilicen, estando en pro y en contra de la cantidad de movimientos del modelo respectivamente.

El propósito de la primera parte de este trabajo es mostrar un punto intermedio que permite animar en forma creíble la manera en que podría caminar un robot con características similares a un ser humano (brazos, piernas, etc.), basándose en la observación del cuerpo humano y la intuición.

En la segunda parte se extiende este punto intermedio hacia un modelo con más movilidad, sin abandonar la representación simple de brazos, piernas, etc. De este modo el movimiento se aplica a este modelo para luego reemplazar las partes representadas por figuras más depuradas y semejantes a las humanas.
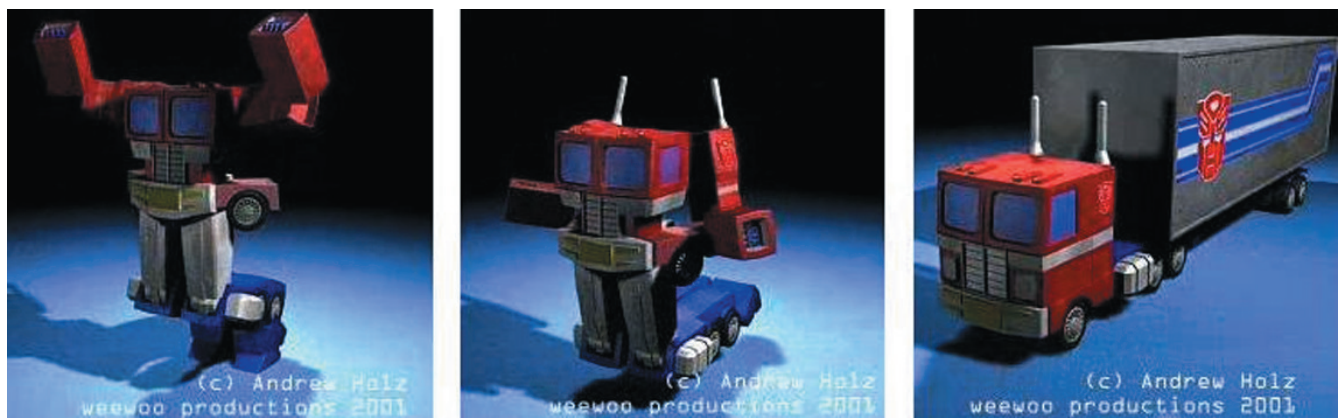

Fig. 2 Secuencia de transformación

En la Figura 1 [6] podemos apreciar la similitud del robot con la anatomía humana, distinguiendo brazos, piernas, pecho, cabeza, etc. Cada una de estas partes se modela por separado. Como se ve en la Figura 2[3], la secuencia de transformación muestra en forma clara 

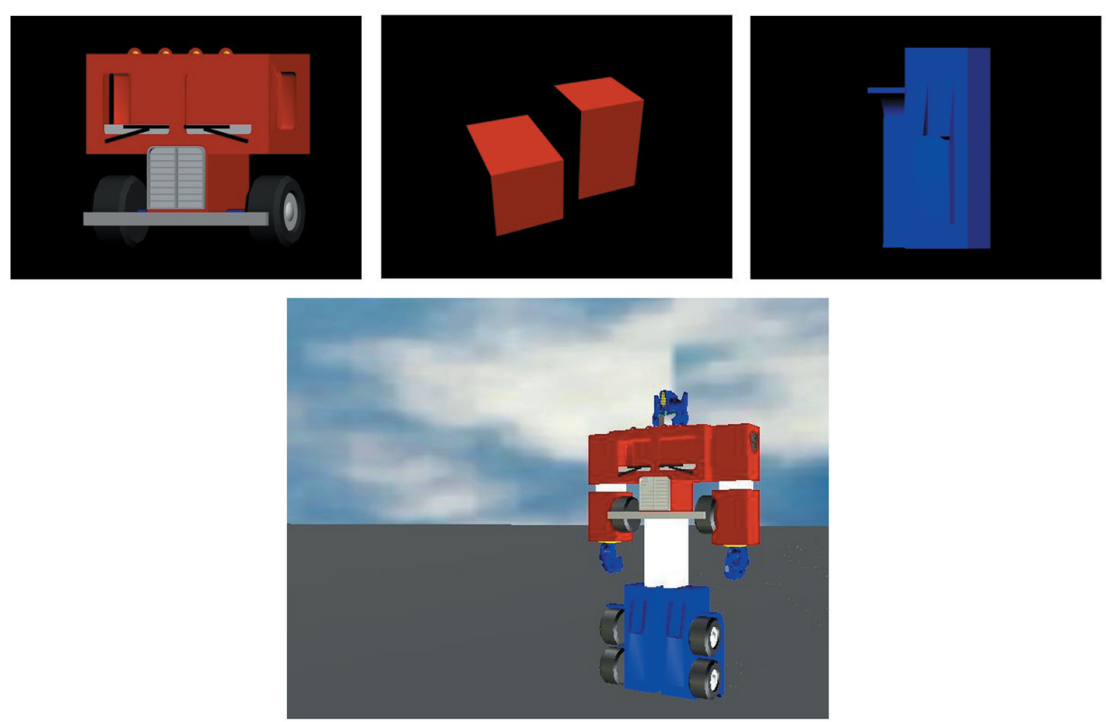

Fig. 3 Arriba, de izquierda a derecha: pecho, brazo y pantorrilla. Abajo: el robot completo.

las partes que participan en la transformación, y en qué forma encajan para transformarse en camión. También podemos ver que el modelo requiere de movimientos en los brazos y piernas manteniendo fijo el pecho; a su vez, los brazos se mueven desde los hombros permitiendo el movimiento independiente del antebrazo en torno al codo, por otro lado, las piernas se mueven en torno a la cintura permitiendo el movimiento independiente de las pantorrillas en torno a las rodillas. Luego de ver las imágenes y teniendo en cuenta que los movimientos para caminar serán similares a los de una persona, las piezas independientes a modelar y dotar de movimiento son las siguientes: Pierna, se compone de pantorrilla y muslo; Brazo, se compone de antebrazo, codo y hombro y la Cabeza, que se esconde en el pecho.

El Pecho y las Ruedas no tienen movimientos independientes, por lo que se pueden modelar en una sola pieza. Las manos no tienen una importancia relevante en el modelo, por lo que son de libre implementación.

En la Figura 3 se pueden ver las piezas principales por separado y el modelado final del robot. La anatomía del robot conserva la similitud con el original y sobre todo con la humana, resumiendo las capacidades de movimiento sólo al de brazos, piernas y del robot completo.

La unión de estas partes puede ser vista como un árbol, en la que cada nodo representa una parte del robot que a su vez se compone de sus nodos hijos, así la raíz del árbol representa al robot completo; en la Figura 4 se puede ver una idea de lo anterior. Luego en la animación veremos cómo esta visión facilita el movimiento del robot ya que si queremos mover la pierna izquierda (piernaI en la figura 4) sólo movemos el nodo correspondiente y se moverán tanto el muslo como la pantorrilla.

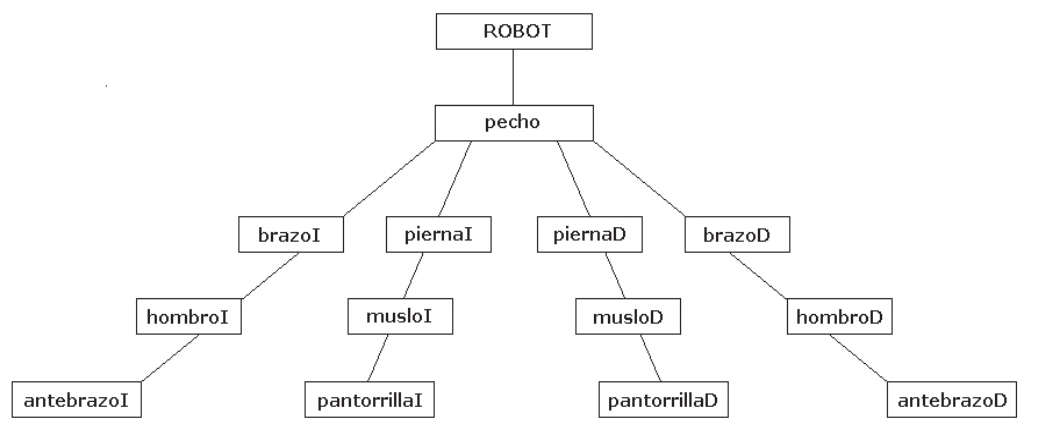

Fig. 4 Diagrama de unión de la piezas. Las I's y D's al final de cada nombre en los nodos son para diferenciar si corresponden al lado izquierdo I o derecho D del robot. 

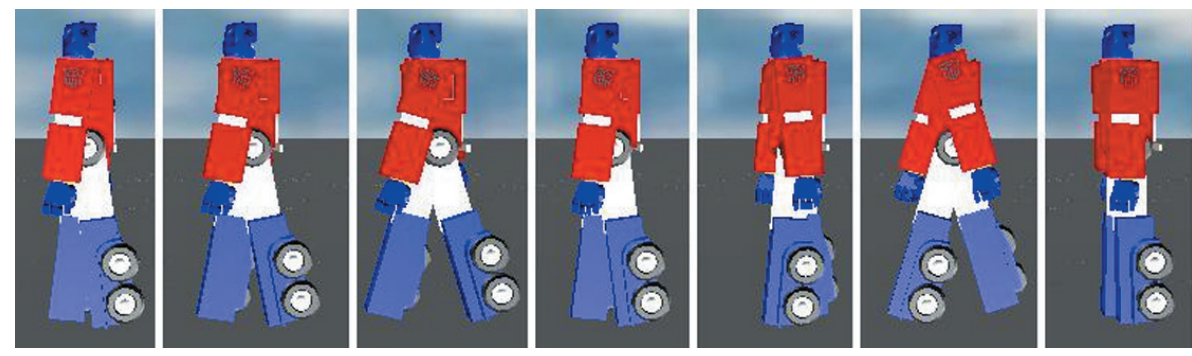

Fig. 5 Secuencia principal de desplazamiento.
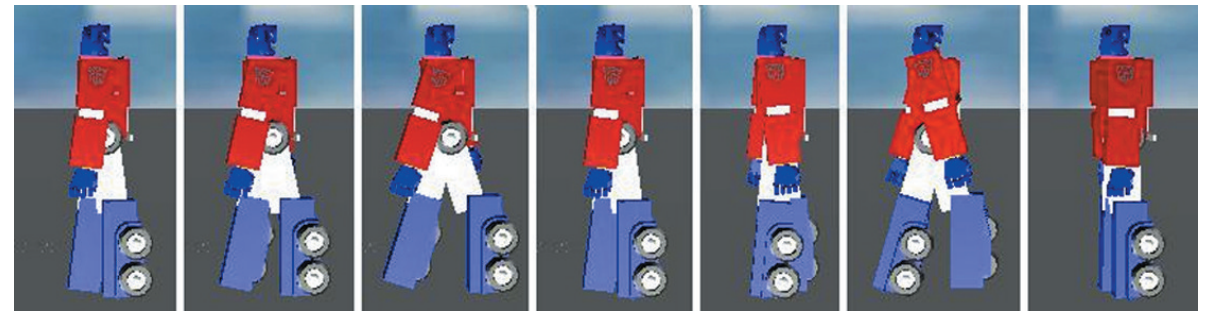

Fig. 6 Secuencia con el movimiento de pantorrilla incluido.

\section{Animación del modelo}

Sin duda, la animación del modelo es la parte más difícil de implementar; esta se dividió en tres partes: robot, secuencia de transformación y camión. Para lograr los movimientos, se definieron secuencias de posiciones para cada parte según la iteración del movimiento.

\section{-Animación del robot}

El primer paso a seguir es crear una secuencia de movimientos para las extremidades; esta secuencia se realiza para los nodos piernaI, piernaD, brazoI y brazoD de la Figura 4 y consiste en mover en forma similar a un péndulo pero con velocidad constante cada extremidad. Estos movimientos deben ser iguales tanto en amplitud máxima como velocidad, además el brazo y pierna del mismo lado deben moverse en sentidos distintos, es decir, la piernaI debe moverse en sentido contrario a la piernaD similar a los movimientos de una tijera. Esto genera movimientos cruzados, la piernaI y el brazoD oscilan de la misma forma, pero por otro lado la piernaD y el brazoI oscilan en el sentido contrario. Esta primera secuencia es la principal para crear la sensación de desplazamiento del robot. En la Figura 5 se muestra la secuencia de la primera etapa del movimiento de las extremidades.

El siguiente paso es dotar de movimiento a los nodos pantorrillaI y pantorrillaD de la Figura 4 con el propósito de ver una flexión en las rodillas. Para esto se debe mover la pantorrilla de la misma forma que la pierna, pero en sentido contrario a partir del momento en que la pierna pasa a oscilar hacia delante. Como la pantorrilla ya se encontraba oscilando (por herencia de su nodo padre) en un sentido, al hacer que la pantorrilla oscile en sentido contrario se logra que ésta se mantenga perpendicular al suelo cuando pasa hacia adelante, de forma que se logra dar la sensación de apoyo y estabilidad al caminar. Para agregar una sensación de esfuerzo o tracción, el eje de oscilación de las pantorrillas es distinto al de unión con el muslo, este último se sitúa más atrás que el eje de oscilación, de esta forma el ir avanzado da la impresión de que el muslo se monta sobre la pantorrilla. En la Figura 6 se puede apreciar este movimiento, en esta se puede ver que la unión del muslo con la pantorrilla no se realiza sobre un punto simétrico, sino que casi se realiza en la punta de la pantorrilla, en cambio el eje de oscilación de ésta se encuentra en un punto por delante de la unión con el muslo. Además se aprecia que cuando la pierna comienza su trayectoria hacia adelante la pantorrilla se mantiene perpendicular al piso.
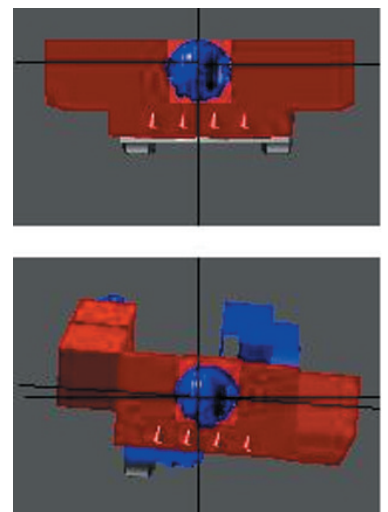

Fig. 7 Oscilación del robot en torno a su eje de simetría. 

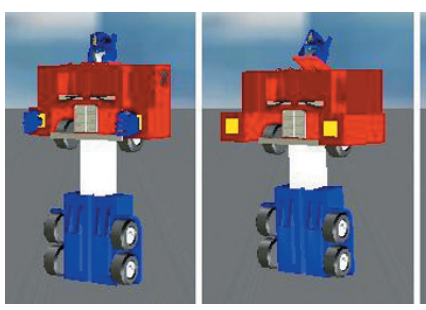
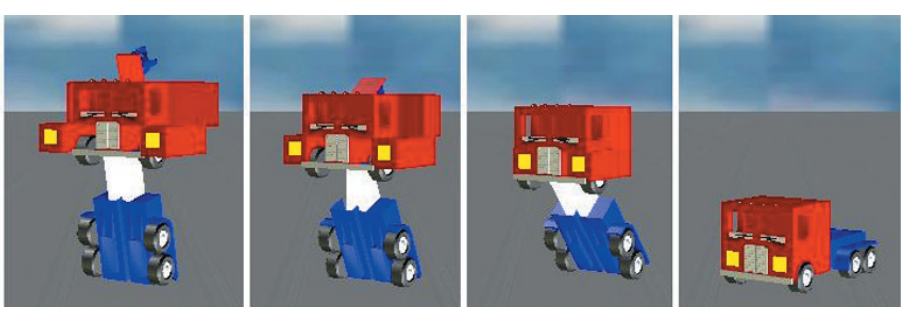

Fig. 8 Secuencia de transformación.
El siguiente paso es dotar de movimiento al nodo pecho de la Figura 4, que corresponde a hacer oscilar esta pieza en torno a un eje perpendicular al piso, correspondiente a su eje de simetría. El movimiento de esta pieza permitirá dar una sensación de balanceo e impulso al desplazamiento del robot; en la Figura 7 se aprecia cómo el pecho (y todos sus nodos hijos) oscila en torno a su eje de simetría.

Por último, se debe mover todo el robot, para lo cual se debe dotar de movimiento al nodo ROBOT de la Figura 4. El movimiento de este nodo es relativamente simple, ya que sólo corresponde al desplazamiento del robot sobre la superficie, desplazamiento que es continuo y se realiza a la misma velocidad de oscilación de las piernas; esto permite que la pierna que se encuentra delante mantenga la pantorrilla fija de modo que dé la impresión que está ejerciendo la tracción necesaria para caminar y que no está resbalando sobre el suelo.

\section{-Animación de la secuencia de transformación}

Esta secuencia corresponde a una aproximación de los movimientos vistos en la Figura 2. Esta secuencia se aprecia en la Figura 8. La parte más interesante de esta secuencia corresponde al movimiento de los antebrazos; al igual que las pantorrillas al caminar, éstos giran en dirección contraria al movimiento de rotación del brazo, lo que da la impresión de que permanecen estáticos respecto a los hombros. El movimiento de las piernas se limita a girarlas 90 grados en torno al pecho y el de la cabeza a girarla hacia atrás.

\section{-Animación del camión}

Esta animación corresponde a desplazar en forma recta y continua al camión; no se implementó otra clase de movimientos ya que el objetivo principal es hacer caminar al robot, por esta razón no se profundizó en esta etapa.

\section{Resultados de la animación del robot}

Una vez que se implementó el modelo, se aprecia que el robot camina en forma continua y armónica. Los movimientos desarrollados para las rodillas, brazos y para el robot completo cumplen satisfactoriamente el objetivo de que el movimiento al caminar sea creíble. El robot muestra una sensación de realizar esfuerzo al caminar y que la tracción con el piso es continua sin dar paso a efectos de resbalamiento o a flotar sobre el suelo. La animación del modelo descrito puede ser construida a partir de un ángulo dado para la amplitud del movimiento de las piernas y la velocidad de desplazamiento.

\section{ANIMACIÓN DE FIGURA HUMANA}

Basado en la parte anterior, en ésta se describe el método desarrollado para animar una figura humana en forma rápida y fácil. $\mathrm{El}$ método propuesto se divide en dos partes:

- Creación de los cuerpos: Se describen dos pasos a seguir: la creación de un modelo generalizado en el cual se construye el cuerpo con líneas rectas representativas de cada parte a modelar y la creación de un modelo específico, que consiste en cambiar la líneas representativas por figuras depuradas del cuerpo humano.

- Animación: Una vez construido el cuerpo, se pueden aplicar las heurísticas desarrolladas en la primera parte. Por razones de costo computacional es preferible probar el método (en una primera instancia) sobre el modelo generalizado.

\section{Creación de los cuerpos}

\section{- Modelo generalizado}

En esta parte se creará el cuerpo humano con líneas representativas de cada parte, con el propósito de modelarlo usando proporciones o medidas particulares prescindiendo del detalle físico del cuerpo. 
Siguiendo con la visión de árbol planteada en la primera parte, el método de construcción es el siguiente:

1. Dividir el cuerpo en dos partes, tren superior y tren inferior.

2. Definir las líneas que representan cada parte a modelar, es decir, qué partes se modelarán.

3. Unir las líneas, definiendo como pivote para la parte anexada el punto de unión.

4. Unir el tren superior con el inferior.

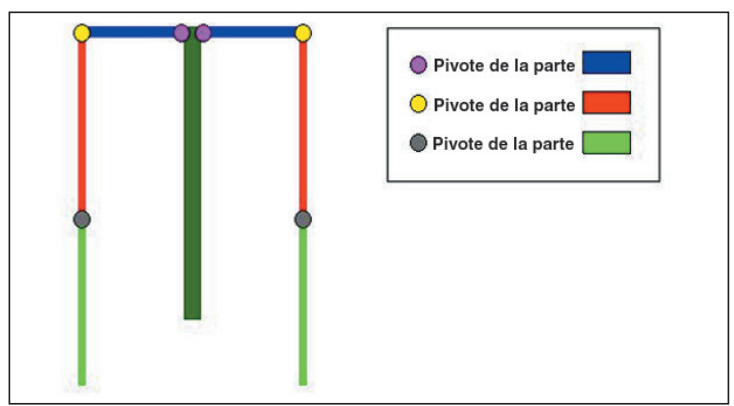

Fig. 9 Visualización y correspondencia de los puntos pivotes.

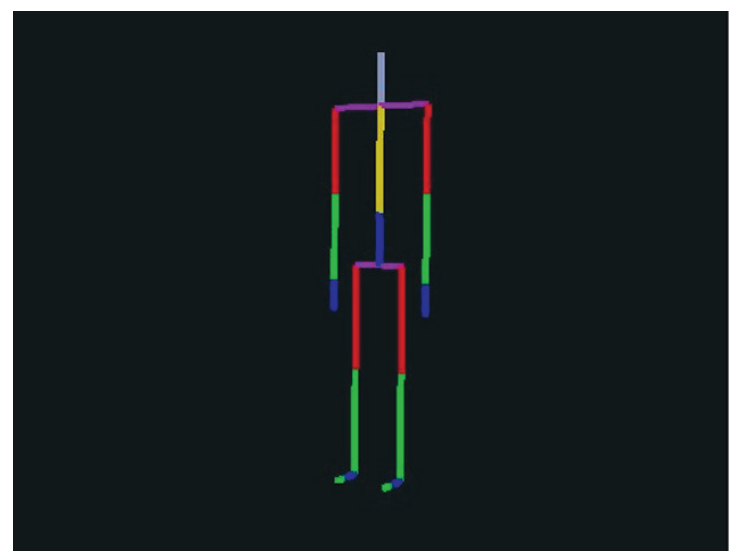

Fig 10 Modelo generalizado.

En la Figura 9 se aprecia parte de la construcción del tren superior de una figura humana, donde se observa la condición de pivote de cada unión. Cada parte puede rotar en torno al pivote sobre cualquier eje; luego en la parte animación crearemos restricciones que impidan sobrepasar ángulos imposibles de realizar por alguna extremidad. En la Figura 10 se aprecia el modelo generalizado de un cuerpo humano; las partes representadas se pueden diferenciar por el color.

\section{- Modelo específico}

Teniendo el modelo generalizado, basta con cambiar cada línea por la forma que se desee, mientras se siga siendo consistente con las medidas y proporciones. En la Figura 11 se aprecia el reemplazo de algunas partes y en la Figura 12 dos modelos terminados. En esta etapa se puede usar la heurística usada en la primera parte, respecto al pivote de las canillas. Para esto es necesario que la figura que reemplazará a la línea que representa la canilla no esté centrada en torno a ella. Así se consigue que la rodilla (pivote) realice un pequeño movimiento al flectarse.

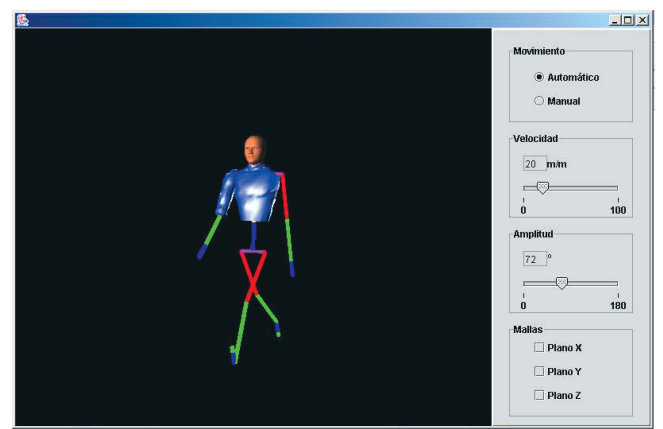

Fig. 11 Modelo específico en construcción.

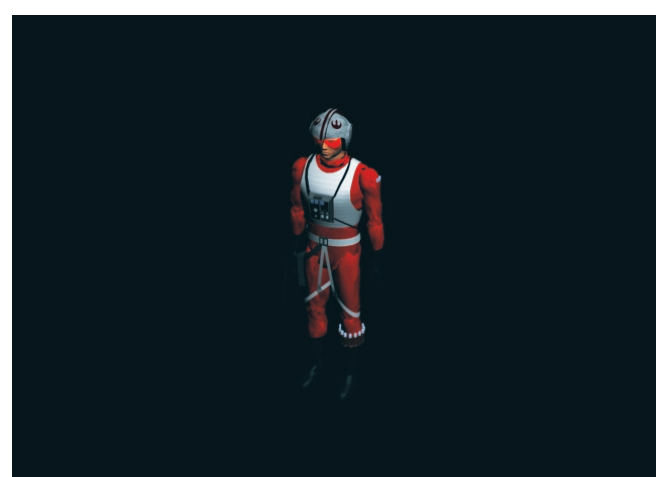

Fig. 12 Modelo específico.

\section{Animación}

Como se mostró en la primera parte de este informe, los movimientos seguían una secuencia de puntos bien definidos para una amplitud y velocidad dadas. Por esta razón, el propósito de esta parte es encontrar una fórmula en función de estas variables y evaluar la función con un factor en particular para cada parte movible.

Debido al movimiento periódico de las extremidades, se usó una ecuación de movimiento armónico simple, para simular los movimientos. La función es la siguiente:

$$
f(A, v)=\text { factor } \cdot A \cdot(\text { eje }+ \text { tipo } \cdot \operatorname{sen}(v \cdot i-\operatorname{arcsen}(\text { eje })))
$$


tipo : $\{-1,1\}$, determina si corresponde al lado izquierdo (-1) derecho (1). Sólo afecta el sentido de la oscilación.

factor : corresponde al factor de la parte a mover

A : amplitud de las piernas

eje : ángulo de inclinación del eje de la oscilación

$\boldsymbol{v} \quad$ : velocidad

$i \quad$ : grado de iteración, se relaciona con el número de cuadros por segundo

En la Figura 13 se puede ver la representación gráfica de la fórmula obtenida. En ella se aprecia en color azul la curva generada por la función seno, luego a esta función se le agrega un desplazamiento sobre el eje y con esto se consigue un movimiento más amplio en algún sentido cuando se desee (curva de color verde en la Figura 13). Por ejemplo, con respecto al movimiento de las piernas, la rodilla se levanta más hacia adelante que hacia atrás, esto se puede apreciar en la Figura 14. Después de aplicar ese desplazamiento, es necesario corregir el origen de la ecuación, ya que de no hacerlo la posición de descanso (amplitud 0) serían las piernas hacia delante y no verticales en el caso de las piernas descrito anteriormente.

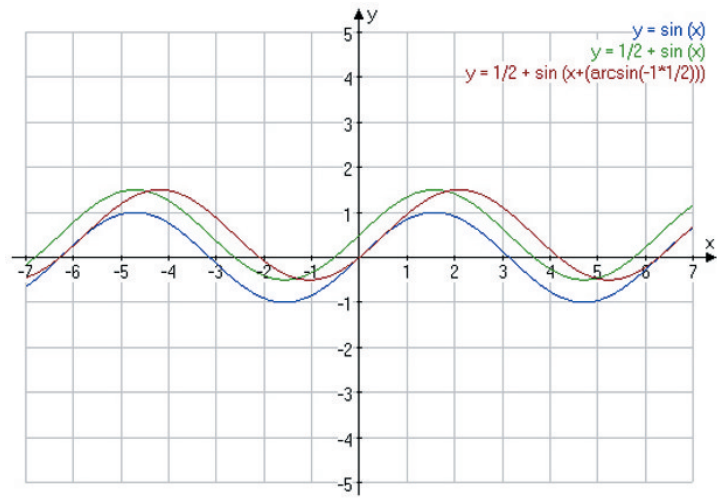

Fig. 13 Representación gráfica.

Pues bien, con esta función ya se pueden generar los movimientos oscilatorios de las partes del cuerpo. A continuación se muestra como se usó esta función para animar el movimiento de las piernas de la Figura 14.

El movimiento de las piernas se divide en dos partes, muslo y canilla (para efectos ilustrativos no se considerará el de los pies). En la Figura 14 se aprecian estos movimientos. El movimiento de los muslos es la evaluación directa de la fórmula para una amplitud de $123^{\circ}$.
El movimiento de las canillas se divide en tres partes, estas son:

1. El factor de oscilación es 0,7 (0,7 veces la amplitud de los muslos).

2. Restricción: en ningún momento el ángulo de oscilación debe ser menor a cero. Es decir, la canilla no se puede doblar por sobre la rodilla.

3. Cuando el ángulo de oscilación es cero (la pierna está estirada en forma vertical), el sentido de la oscilación cambia.

Con esto se logra mantener flexionada la rodilla. Para esto se necesita evaluar la ecuación de dos formas distintas según el ángulo de oscilación.

Para el movimiento de las otras partes es necesario realizar restricciones o condiciones similares según el criterio de quien esté realizando la animación. Por ejemplo, una restricción natural es que el ángulo de oscilación de los antebrazos no sea menor a cero; al igual que en el caso de las rodillas, un ser humano no puede flectar el antebrazo por detrás de la articulación del codo.

\section{Resultado de la animación humana}

Tanto el método desarrollado para la construcción de las figuras como la función creada para mover las partes son métodos alternativos para lograr animaciones creíbles en forma rápida y fácil. El nivel de detalle del cuerpo a modelar sólo depende de la cantidad de líneas o partes representadas en el modelo generalizado, siendo su animación tan generalizable como se desee, imponiendo restricciones y condiciones al movimiento de cada parte.

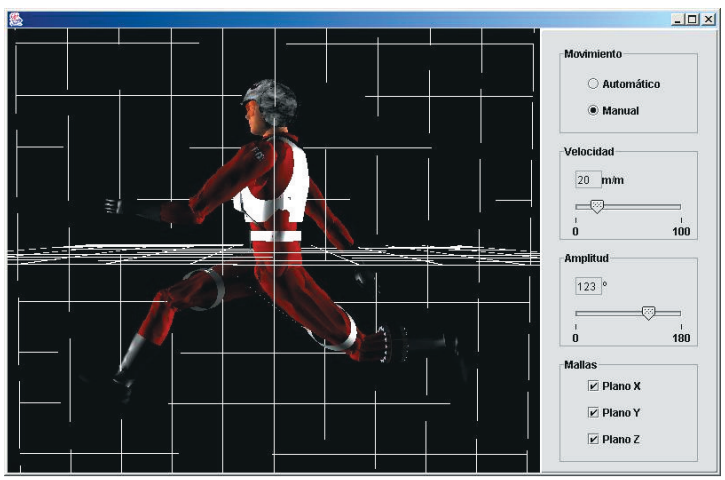

Fig. 14 Animación del movimiento de las piernas. 


\section{CONCLUSIONES}

El método descrito en este trabajo propone una manera simple y rápida de crear y animar figuras humanas. La independencia de las componentes físicas (como la masa) de las figuras permiten aplicar el método con facilidad sobre figuras reales o ficticias, permitiendo la generación de nuevos caracteres y su animación con facilidad ya que no requiere de métodos más sofisticados como en otros modelos convencionales de animación o más avanzados como "motion capture", que además de requerir una mayor cantidad de información necesitan una infraestructura y hardware específicos.

La separación entre los modelos generalizado y específico permite que la definición de los movimientos sea tan detallada como se desee. Se pueden crear tantas restricciones como se estime conveniente, o dividir en más partes la figura, aumentando el detalle de la forma como de la animación.

\section{REFERENCIAS}

[1] Alan Watt and Mark Watt. "Advanced Animation and Rendering Techniques". Theory and Practices. New York, Addison Wesley Pub Company.

[2] Joseph Laszlo, Michiel van de Panne, Eugene Fiume. "Interactive Control for Physically - Based Animation", en Computer Graphics Proceedings, Annual Conference Series, 2000.

[3] Reeves, W. T. "Inbetweening for Computer Animation Utilizing Moving Point Constaints" SIGGRAPH 81, 263-269.

[4] Jessica K. Hodgins and Nancy S. Pollard. "Adapting Simulated Behaviors For New Characters", en Computer Graphics (SIGGRAPH 97 Proceedings), pp. 153-162, Addison Wesley, agosto de 1997.

[5] J. D. Foley, A. van Dam, S.K. Feiner and J.F. Hughes. "Computer Graphics Principles and Practice", Second Edition, Addison Wesley Pub Company, 1990.

[6] Imágenes creadas por Andrew Holz (aholz@hotkey.net.au),weewoo productions 2001. 